\begin{tabular}{l}
$\begin{array}{l}\text { Operators } \\
\text { and } \\
\text { Matrices } \\
\text { Volume 3, Number 2 (2009), 261-270 }\end{array}$ \\
\hline
\end{tabular}

\title{
HYPERINVARIANT, CHARACTERISTIC AND MARKED SUBSPACES
}

\author{
Pudji Astuti And Harald K. WimMer
}

\begin{abstract}
Let $V$ be a finite dimensional vector space over a field $K$ and $f$ a $K$-endomorphism of $V$. In this paper we study three types of $f$-invariant subspaces, namely hyperinvariant subspaces, which are invariant under all endomorphisms of $V$ that commute with $f$, characteristic subspaces, which remain fixed under all automorphisms of $V$ that commute with $f$, and marked subspaces, which have a Jordan basis (with respect to $f_{\mid X}$ ) that can be extended to a Jordan basis of $V$. We show that a subspace is hyperinvariant if and only if it is characteristic and marked. If $K$ has more than two elements then each characteristic subspace is hyperinvariant.
\end{abstract}

Mathematics subject classification (2000): 15A18, 47A15, 15A24, 15A57, 13 C99.

Keywords and phrases: hyperinvariant subspaces, marked subspaces, characteristic subspaces, invariant subspaces, Jordan basis.

\section{REFERENCES}

[1] P. Astuti And H. K. Wimmer, Regular submodules of torsion modules over a discrete valuation domain, Czechoslov. Math. J., 56 (2006), 349-357.

[2] P. Astuti AND H. K. Wimmer, A class of marked invariant subspaces with an application to algebraic Riccati equations, Automatica, 42 (2006), 1503-1506.

[3] L. Baratchart, P. Enqvist, A. Gombani, And M. Olivi, Surface acoustic wave filters, unitary extensions and Schur analysis, Report No. 44, 2003, Institut Mittag-Leffler, Stockholm, http://www.mittag-leffler.se/preprints/0203s.

[4] R. BRU, L. RodmAn, AND H. SCHNEIDER, Extensions of Jordan bases for invariant subspaces of a matrix, Linear Algebra Appl., 150 (1991), 209-226.

[5] A. COMPtA AND J. FerReR, On $(A, B)^{t}$-invariant subspaces having extendible Brunovsky bases, Linear Algebra Appl., 255 (1997), 185-201.

[6] A. COMPTA, J. Ferrer, AND M. PeÑA, Dimensions of the orbit of marked subspaces, Linear Algebra Appl., 379 (2004), 239-248.

[7] A. Compta, ANd M. PEÑA, Structural stability of $(C, A)$-marked subspaces, Linear Algebra Appl., 421 (2007), 45-52.

[8] J. Ferrer, F. Puerta, And X. Puerta, Geometric characterization and classification of marked subspaces, Linear Algebra Appl., 235 (1996), 15-34.

[9] P. A. Fillmore, D. A. Herrero, And W. E. LongstafF, The characteristic subspace lattice of a linear transformation, Linear Algebra Appl., 17 (1977), 125-132.

[10] L. Fuchs, Infinite Abelian Groups, Vol. I., Academic Press, New York, 1973.

[11] F. R. Gantmacher, The Theory of Matrices, Vol. I, Chelsea, New York, 1959.

[12] I. Gohberg, P. LANCASter, And L. Rodman, Matrices and Indefinite Scalar Products, Operator Theory: Advances and Applications, Vol. 8, Birkhäuser, Basel, 1983.

[13] I. Gohberg, P. Lancaster, And L. Rodman, Invariant Subspaces of Matrices with Applications, Wiley, New York, 1986.

[14] I. KaPlans Ky, Infinite Abelian Groups, University of Michigan Press, Ann Arbor, 1954.

[15] T. KošIR AND B. PlestenJAK, On stability of invariant subspaces of commuting matrices, Linear Algebra Appl., 342 (2002), 133-147.

[16] V. KUČERA, Algebraic Riccati equations: Hermitian and definite solutions, in S. Bittanti, A. J. Laub and J. C. Willems (Eds.), The Riccati Equation, 53-88, Springer Verlag, Berlin, 1991. 
[17] W. E. LONGSTAF, A lattice-theoretic description of the lattice of hyperinvariant subspaces of a linear transformation, Can. J. Math., 28 (1976), 1062-1066.

[18] L. Rodman, Maximal invariant neutral subspaces and an application to the algebraic Riccati equation, Manuscr. Math., 43 (1983), 1-12.

[19] P. Y. WU, Which linear transformations have isometric hyperinvariant subspace lattices?, Linear Algebra Appl., 169 (1992), 163-178. 